\title{
Multiscale entropy analysis of electroseismic time series
}

\author{
L. Guzmán-Vargas ${ }^{1,3}$, A. Ramírez-Rojas ${ }^{2}$, and F. Angulo-Brown ${ }^{3}$ \\ ${ }^{1}$ Unidad Profesional Interdisciplinaria en Ingeniería y Tecnologías Avanzadas, Instituto Politécnico Nacional, Av. IPN No. \\ 2580, Col. Ticomán, México D. F. 07340, Mexico \\ ${ }^{2}$ Departamento de Ciencias Básicas, Universidad Autonoma Metropolitana, Av. San Pablo 180, Col. Reynosa, Azcapotzalco, \\ México D. F. 02200, Mexico \\ ${ }^{3}$ Departamento de Física, Escuela Superior de Física y Matemáticas, Instituto Politécnico Nacional, Edif. No. 9 U.P. \\ Zacatenco, México D. F. 07738, Mexico
}

Received: 5 June 2008 - Revised: 8 July 2008 - Accepted: 8 July 2008 - Published: 15 August 2008

\begin{abstract}
In this work we use the multiscale entropy method to analyse the variability of geo-electric time series monitored in two sites located in Mexico. In our analysis we consider a period of time from January 1995 to December 1995. We systematically calculate the sample entropy of electroseismic time series. Important differences in the entropy profile for several time scales are observed in records from the same station. In particular, a complex behaviour is observed in the vicinity of a $M=7.4$ EQ occurred on 14 September 1995. Besides, we also compare the changes in the entropy of the original data with their corresponding shuffled version.
\end{abstract}

\section{Introduction}

Nowadays, the efforts to develop earthquake prediction methods, have not been succesful. However, despite some pressimism, in many seismically actives zones around the world there exist research programs for the study of possible precursory phenomena of eathquakes (Lomnitz, 1990; Hayakawa, 1999; Hayakawa and Molchanov, 2002; Telesca et al., 2005). In particular, one of the techniques used in the search of earthquake precursors since more than three decades ago consists in monitoring the so-called electric selfpotential field. This was motivated by the following aspects (Varotsos, 2005): It is expected that before an earthquake occurrence, the stress (pressure) gradually varies in the focal area, which affects various physical properties, for example the static dielectric constant (Varotsos, 1978, 1980). In addition, this stress variation may change the relaxation time for the orientation of the electric dipoles formed due to lattice defects (Lazaridou et al., 1985). It may happen that,

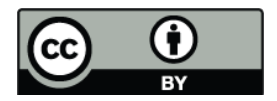

Correspondence to: L. Guzmán-Vargas (lguzmanv@ipn.mx) when the stress (pressure) reaches a critical value (Varotsos and Alexoupoulos, 1984b), these electric dipoles exhibit a cooperative orientation (collective organization), thus leading to emission of transient electric signals termed Seismic Electric Signals, SES. Hence, since criticality is always associated with fractality, it is expected that precursory electric signals should exhibit fractal properties (Varotsos, 2005). We have measured the ground electrical potential (the selfpotential) in several sites along the Mexican coast, near the Middle American trench, which is the border between the Cocos and the American tectonic plates. In some previous articles we have reported more detailed descriptions of that region and some studies of possible precursory electric phenomena associated to several earthquake of magnitude larger than six (Muñoz-Diosdado et al., 2004; Ramírez-Rojas et al., 2007; Flores-Márquez et al., 2007). Recent studies focused on fractal and non linear properties of physical and biological times series have revealed that this organization is strongly related to a complex interaction of multiple components and mechanims across multiple scales. In particular, published studies about the complexity of ground electric self-potential behavior have pointed out that changes in the fractal organization have been observed in a period prior to an important earthquake. However, a clear evidence with statistical support about the mechanisms involved in these changes, has not been presented, although some important suggestions and discussions have been proposed to address this problem (Gotoh et al., 2003, 2004; Smirnova et al., 2004; Ida et al., 2005; Ida and Hayakawa, 2006; Telesca and Lapenna, 2006; Varotsos et al., 2008). One important feature of geolectric signals is the asbsence of regularity patterns when a time series is observed by means of traditional methods. The direct application to this kind of signals of nonlinear methods such as power spectrum, detrended fluctuation analysis (DFA) and fractal dimension method reveals that different correlation levels are present in the vicinity of a big event. It is important

Published by Copernicus Publications on behalf of the European Geosciences Union. 
to apply different kinds of methods to the same data, to confirm and make sure that something is really taking place prior to a big earthquake.

In this work, we present a study to evaluate the changes in the variability of geolectric signals during a one year period from 1 January to 31 December 1995 in two sites located in the Guerrero coast in southern Mexico. In particular, our study is related to an $M s=7.4$ earthquake ocurred on 14 September 1995. Previous studies by our group have reported changes in the correlation dynamics observed prior to this earthquake (Ramírez-Rojas et al., 2007, 2004). We use the sample entropy algorithm to evaluate the level of regularity of geolectric time series across multiple time scales. A similar study, but with a different entropy defined (Varotsos et al., 2003) in a new time domain termed natural time, has been made by Varotsos et al. (2004), to distinguish SES activities from signals emitted from man-made sources. We observe important changes in the irregularity of the signals for a period in the vicinity of the earthquake mentioned above. The results presented here are consistent with previous studies of the same data based on power spectral analysis and fractal dimension methods (Ramírez-Rojas et al., 2004). The paper is organized as follows. In Sect. 2, a brief description of the approximated entropy and sample entropy methods are presented. We also describe the electroseismic time series. In Sect. 3, we present the results and discussions. Finally in Sect. 4, some concludings remarks are given.

\section{Data and methods}

\subsection{Entropy methods}

The entropy of a single discrete random variable $X$ is a measure of its uncertainty. In the Shannon's entropy definition, the average uncertainty of a discrete random variable that obtains values from a finite set $x_{1}, \ldots, x_{n}$, with probabilities $p_{1}, \ldots, p_{n}$, is given by

$H(X)=-\sum_{x_{i}} p\left(x_{i}\right) \log p\left(x_{i}\right)$,

where $X$ is the random variable and $p\left(x_{i}\right)=P_{r}\left(X=x_{i}\right)$ is the probability mass function. In the case of a stochastic process, the mean rate of creation of information is known as the Kolmogorov-Sinai (KS) entropy (Eckmann and Ruelle, 1985). However, the KS definition is not applicable to finite length real world series because numerically only entropies of finite order can be computed and $\mathrm{KS}$ is understimated as the order becomes large. An alternative procedure to estimate the entropy of a signal was given by Grassberger and Procaccia (Grassberger and Procaccia, 1983). They proposed the $K_{2}$ entropy to characterize chaotic systems which is a lower bound of the $K S$ entropy. Later, based on $K_{2}$ definition, Pincus introduced the Approximate Entropy ( $A p E n$ ) to quantify the regularity in time-series (Pincus, 1991, 1995). Briefly, ApEn is constructed as follows: given a time series $X_{i}=x_{1}, \ldots, x_{N}$ of length $N$. First, $m$-length vectors are considered: $u_{m}(i)=$ $x_{i}, x_{i+1}, \ldots, x_{i+m-1}$. Let $n_{i m}(r)$ represent the number of vectors $u_{m}(j)$ within $r$ of $u_{m}(i) . C_{m}^{i}(r)=n_{i m}(r) /(N-$ $m+1)$ is the probability that any vector $u_{m}(j)$ is within $r$ of $u_{m}(i)$. Next, the average of $C_{m}^{i}$ is constructed, $\Phi^{m}(r)=$ $1 /(N-m+1) \sum_{i=1}^{N-m+1} \ln C_{i}^{m}(r)$. Finally, ApEn is defined as $\operatorname{ApEn}(m, r)=\lim _{N \rightarrow \infty}\left[\Phi^{m}(r)-\Phi^{m+1}(r)\right]$. For finite $\mathrm{N}$, it is estimated by the statistics, $\operatorname{ApEn}(m, r, N)=$ $\Phi^{m}(r)-\Phi^{m+1}(r)$. In words, the statistics $\operatorname{ApEn}(m, r, N)$ is approximately equal to the negative average natural logarithm of the conditional probability that two sequences that are similar for $m$ points remain similar at the next point, within a tolerance $r$ (Richman and Moorman, 2000). Then, a low value of $A p E n$ reflects a high degree of regularity. Even though the implementation and interpretation of $A p E n$ is useful to distinguish correlated stochastic processes and composite deterministic/stochastic models (Pincus, 1995), it has been found there is a bias in $A p E n$ because the algorithm counts each sequence as matching itself (Richman and Moorman, 2000). The presence of this bias causes ApEn to lack two important expected properties: (a) ApEn is heavily dependent on the time-series length and is uniformly lower than expected for short series and, (b) it lacks relative consistency in the sense that if value of $A p E n$ for a time-series is higher than that of another, it does not remain so if the test conditions change (Pincus, 1995). Therefore, the development of an alternative method was desirable to overcome the limitations of $A p E n$. Based on $K_{2}$ and ApEn methods, Richman and Moorman (Richman and Moorman, 2000) introduced the sample entropy $\left(S_{E}\right)$, to reduce the bias in ApEn. One of the advantages of $S_{E}$ is that it does not count self-matches and it is not based on a template-wise approach (Richman and Moorman, 2000). $S_{E}(m, r, N)$ is precisely defined as

$S_{E}(m, r, N)=-\ln \frac{U^{m+1}}{U^{m}}$

that is, the negative natural logarithm of the conditional probability $(U)$ that two sequences similar for $m$ points remain similar at the next point, within tolerance $r$, without counting the self-matches. $S_{E}$ results would be more robust than $A p E n$ statistics when applied to short time series from different stochastic processes over a wide range of operating conditions. For instance, a lower value of $S_{E}$ indicates a more regular behavior of a time-series wheareas a high value is assigned to more irregular, less predictable, time series. It applies to real world time series and, therefore, has been widely used in physiology and medicine (Costa et al., 2005).

\subsection{Multiscale entropy analysis}

Recently, Costa et al. (2002) introduced the multiscale entropy analysis (MSE) to evaluate the relative complexity of normalized time series across multiple scales. This 


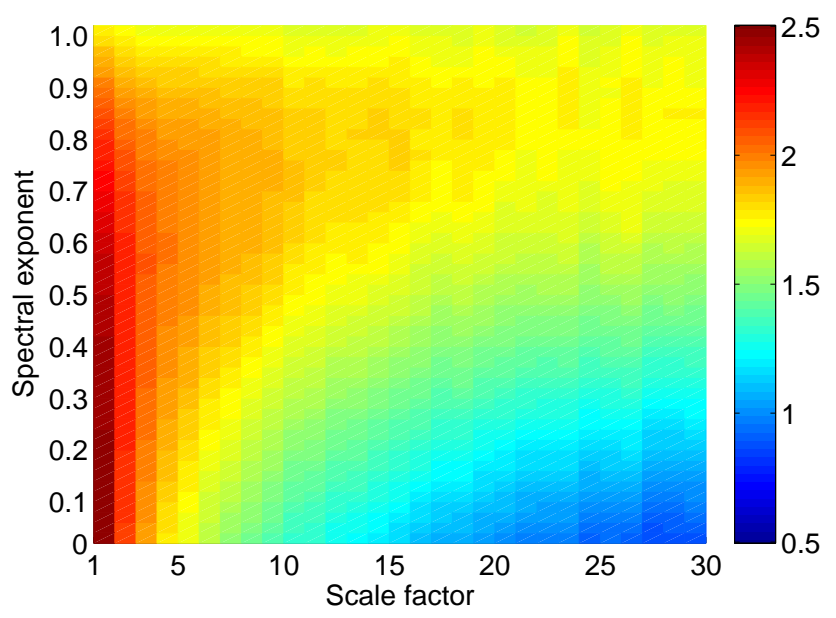

Fig. 1. Plot of MSE analysis for $1 / f^{\beta}$-noises with $0 \leq \beta \leq 1$, that is, for noises with power-law correlations. We used the Fourier filtering method to generate time series of 32000 points. In this plot, each point represents the average of 10 independent realizations. The value of $S_{E}$ is given according to the color panel. Note that as the spectral exponent increases the entropy value remains high even for large time scales.

procedure was proposed to give an explanation to the fact that, in the context of biological signals, single-scale entropy methods ( $S_{E}$ and $A p E n$ ) assign higher values to random sequences from certain pathologic conditions whereas an intermediate value is assigned to signals from healthy systems (Goldberger et al., 2002; Costa et al., 2002). It has been argued that these results may lead to erroneous conclusions about the level of complexity displayed by these systems (Costa et al., 2005). The MSE methodology shows that long-range correlated noises as the output of healthy systems are more complex than uncorrelated signals from some pathologic conditions. Briefly we explain the MSE, given a time series $X_{i}=x_{1}, \ldots, x_{N}$, a coarse-graining procedure is proposed (Costa et al., 2005). A scale factor $\tau$ is introduced to perform a moving average given by $X_{j}=1 / \tau \sum_{i=(j-1) \tau+1}^{j \tau} x_{i}$, with $1 \leq j \leq N / \tau$. Note that the length of the coarse-grained time series is given by $N / \tau$, that is, for scale 1 , one has the original time series. To complete the MSE procedure the $S_{E}$ algorithm is applied to the time series for each scale. Finally, the entropy value is plotted against the scale factor. Typically, under MSE analysis, the entropy values for a random noise monotically decreases whereas for long-range correlated noise $(1 / f$-noise) the entropy remains constant for several scales, indicating that $1 / f$ noise is structurally more complex than uncorrrelated signals (Costa et al., 2005). In order to get a better estimation of entropy values for Gaussian noises with power law correlations, we performed simulations of noises with power spectrum of the form $1 / f^{\beta}$ with $0 \leq \beta \leq 1$. We generated time series with 32000 points by means of the Fourier filtering
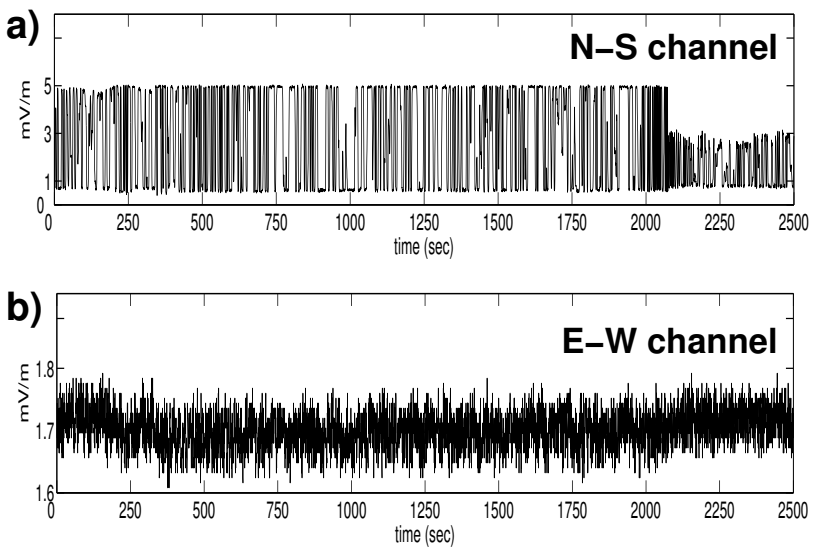

Fig. 2. Representative electroseismic time series from Acapulco station (17 June 1995) (a) N-S channel and (b) E-W channel.

method (Makse et al., 1996). We applied the MSE analysis to the generated data for several values of $\beta$ in the interval $0 \leq \beta \leq 1$ and a range of time scales. In Fig. 1, the results for entropy are presented according to the color panel. Notice that for $\beta=0$ and $\beta=1$, the main results described in Costa et al. (2002) are recovered.

\subsection{Data}

The time series considered in this study were monitored during the year 1995 in two electroseismical stations located at Acapulco $\left(16.85^{\circ} \mathrm{N}, 99.9^{\circ} \mathrm{W}\right)$, and Coyuca $\left(18.35^{\circ} \mathrm{N}\right.$, $\left.100.7^{\circ} \mathrm{W}\right)$, both located in the South Pacific coast in Mexico (Ramírez-Rojas et al., 2004). The electroseismic time series consist of the electric self-potential fluctuations, $\Delta V$, between two electrodes buried $2 \mathrm{~m}$ of depth into the ground and separated $50 \mathrm{~m}$ of distance. Each pair of electrodes was oriented in two directions: North-South, and East-West directions, as it is indicated in VAN methodology (Varotsos and Alexoupoulos, 1984a,b; Varotsos and Alexopoulos, 1987). Two time series were simultaneously recorded at each electroseismic station (N-S and E-W channels). Due to technical adjustments, two different sampling rates were used in different time intervals along the mentioned period, $\Delta t=4 \mathrm{sec}$ onds in Coyuca and $\Delta t=2$ seconds in Acapulco (Yépez et al., 1995). In Fig. 2 representative time series of potential differences for Acapulco station are presented. These potential fluctuations correspond to E-W and N-S channels from a short period of time during June 1995. When we compare these two signals, two different kind of fluctuations can be identified. An important question here is to evaluate the level of irregularity across multiple scales and its relation with the presence of long range correlations. In this work, we evaluate the changes in the variability of these signals during a one year period from 1 January to 31 December 1995 in both stations (Acapulco and Coyuca). 


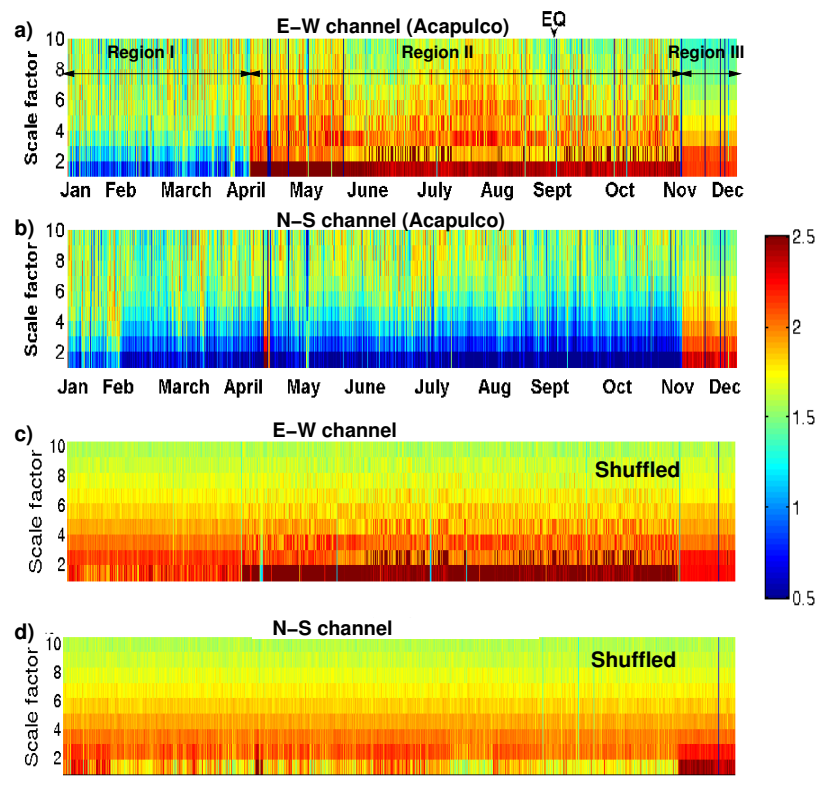

Fig. 3. MSE analysis of geolectrical time series from Acapulco station. (a) MSE results for E-W channel, three main regions can be identified according to the changes of $S_{E}$ for different scale factors. Note that Region II is characterized by a high entropy value even for large time scales. (b) Entropy results for N-S channel. (c), (d) As in (a) and (b) but for randomized data. Note that in these cases the data display mostly white noise profile.

\section{Results and discussion}

For the application of the MSE procedure to the electroseismic time series we considered non overlapped time windows of 3600 data points corresponding to approximately $2 \mathrm{~h}$ of records. First, the original signal is divided by its standard deviation and $S_{E}$ is calculated for each time scale according to the MSE method. We repeated the MSE procedure for the corresponding shuffled version of each window. In all the cases presented here, we used the following parameter values: $r=0.15$ and $m=2$. In Fig. 3, the results of $S_{E}$ for Acapulco station are presented. The color panel represents the values of $S_{E}$ in the interval 0.5 to 2.5 . For E-W channel (Fig. 3a), we observe that for a short period at the beginning of the year (Region I), $S_{E}$ shows a low value for scale one, followed by a small increment for large time scales, that is, a high regularity in the original data is observed. After this period, the entropy value increases and remains high even for scale $\tau=5$, indicating a presence of complex dynamics probably related to long range correlations (Region II). Interestingly, a short period after the EQ 7.4 occurred at 14 September 1995, the entropy value shows a fast decay similar to white noise (Region III). For N-S channel (Fig. 3b), the entropy is small for scale one and shows a small increment as the scale factor increases. This behavior is observed almost for all the year except for a short period at the end where a

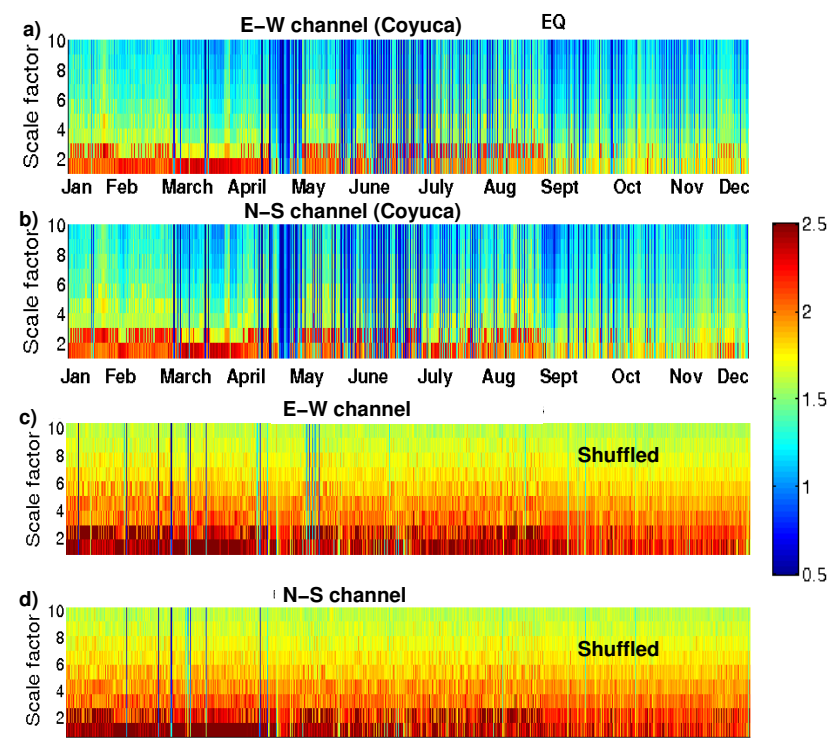

Fig. 4. MSE analysis of geolectrical time series from Coyuca station. (a) MSE results for E-W channel, we observe that entropy value is close to white noise profile except for short periods with a low entropy. (b) Entropy results for N-S channel. (c) and (d) As in (a) and (b) but for randomized data. Note that in these cases the data display mostly white noise profile.

pattern very similar to white noise is present. Previous studies (Ramírez-Rojas et al., 2004), by means of power spectral analysis and fractal dimension methods, indicated the presence of long range correlations in the same period where a complex dynamics is observed by means of the MSE. These studies have reported that from April to September 1995, the power spectrum of E-W time series is described by two spectral exponents, $\beta_{1}$ and $\beta_{2}$ with $\beta_{2} \approx 1$, indicating the presence of long-range correlations. In Fig. $3 \mathrm{c}$ and $\mathrm{d}$ results for the correspoding shuffled versions are presented. For E-W data, we observe that for almost all year a pattern similar to white noise is present, except because a high value, for scale one, is identified in the period of complex dynamics. For N-S channel, the entropy shows a profile similar to white noise.

The results for both channels in Coyuca Station are presented in Fig. 4. We observe that the $S_{E}$ shows a similar profile to white noise except because the presence of mutiple short periods with a low entropy value across multiple scales (see Fig. 4a and b). When these results are compared to their corresponding surrogate sequences, the original profile for the entropy is preserved but the short periods with low entropy values are changed to white noise behavior.

To further evaluate the changes of $S_{E}$ results, we construct scatter plots of entropy values at scale 1 for E-W channel $\left(S_{E}^{E W}(1)\right)$ against entropy for N-S channel $\left(S_{E}^{N S}(1)\right)$. The results are presented in Figs. 5a and 6a. For Acapulco station, we observe that the entropy of the original data is clearly 

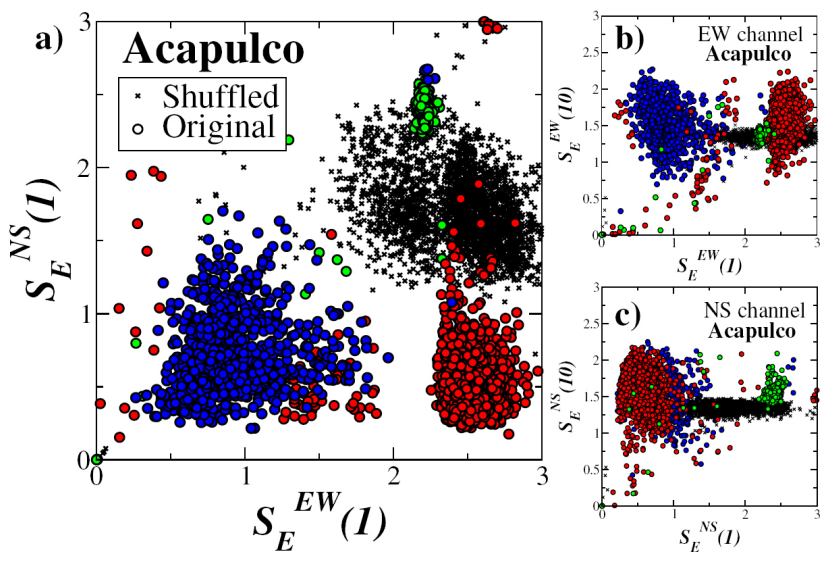

Fig. 5. Scatter plots of $S_{E}$ from MSE analysis for Acapulco station. (a) Entropy from E-W channel vs. N-S channel, for scale 1. The original data (circles) are colored according to the three main regions defined in Fig. 3a (blue (Region I), red (Region II) and green (Region III)). Note that these three regions are clearly segregated. (b) and (c) $S_{E}$ for scale 1 vs. scale 10 .

separated in three main groups corresponding to the three regions defined above. In particular, Region I is characterized by a low entropy value for E-W channel and an intermediate value for N-S. In contrast, Region II is described by a high entropy value for E-W channel and a low entropy for N-S. According to Fig. 5a, Region III shows a strong correlation in entropy values of E-W and N-S channels, that is, the high entropy in both channels resembles white noise behavior. Furthermore, as shown in Fig. 5a the high entropy values of shuffled data in both channels confirm a high variability which resembles white noise. For Coyuca station (Fig. 6a), we observe a clear correlation between entropy values for scale 1, indicating that $S_{E}$ values are positively related. We also observe that results for the shuffled version are very similar to the original data. Additionally, we also construct the corresponding scatter plots to compare the changes in the entropy value for short scales (scale 1) versus large scales (scale 10). These results are described in Figs. 5b, c and 6b, c. For E-W channel in Acapulco station, we observe a good discrimination between the three main time regions described in Fig. 3a whereas for $\mathrm{N}-\mathrm{S}$ channel two main regions can be identified (see Fig. 5b, c). In the case of Coyuca, both channels display a similar behavior which is characterized by a high value at scale 1 and a low value at scale 10 (Fig. 6b, c). For a comparison we also show the data corresponding to the randomized version of each channel.

\section{Conclusions}

We have applied the MSE method to geolectrical signals from two sites in southern Mexico. We have found different entropy values for these signals across several scales. In
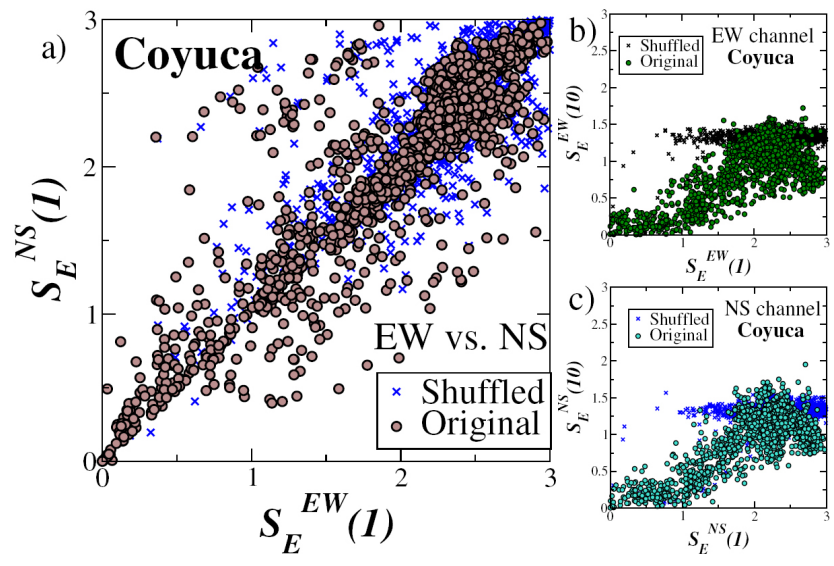

Fig. 6. Scatter plots of $S_{E}$ from MSE analysis for Coyuca station. (a) Entropy from E-W channel vs. N-S channel, for scale 1 . We observe that the entropy values are strongly correlated. (b) and (c) $S_{E}$ for scale 1 vs. scale 10 .

particular, the Acapulco station displays three different patterns of complex dynamics along the year which are clearly identified in E-W channel. The results for Coyuca station reveal that the entropy profile is mostly white noise for both channels. The results of Acapulco station are qualitatively compatible with previous reports based in spectral analysis. Acapulco station was located at approximately $110 \mathrm{~km}$ from the $M=7.4$ epicenter. Coyuca station was located at $200 \mathrm{~km}$ from the epicenter. The MSE method reveals complex fluctuation dynamics for the nearest station (Acapulco) but the results for Coyuca are mainly white noise dynamics. In summary, MSE analysis reveal important information about the complex behavior of these fluctuations and is another important complementary tool in the search of possible geolectric precursory phenomena of earthquakes.

Acknowledgements. L. G.-V. thanks to Area de Física de Procesos Irreversibles, Universidad Autónoma Metropolitana, Mexico. We also thank R. Hernández-Pérez for fruitful suggestions and discussions.

Edited by: M. Contadakis

Reviewed by: two anonymous referees

\section{References}

Costa, M., Goldberger, A. L., and Peng, C.-P.: Multiscale entropy analysis of physiologic time series, Phys. Rev. Lett., 89, 068102, 2002.

Costa, M., Goldberger, A. L., and Peng, C.-K.: Multiscale entropy analysis of biological signals, Phys. Rev. E., 71, 021906, 2005.

Eckmann, J.-P. and Ruelle, D.: Ergodic theory of chaos and strange atractors, Rev. Mod. Phys. 57, 617, 1985.

Flores-Márquez, E. L., Márquez-Cruz, J., Ramírez-Rojas, A., Gálvez-Coyt, G., and Angulo-Brown, F.: A statistical analysis of electric self-potential time series associated to two 1993 earth- 
quakes in Mexico, Nat. Hazards Earth Syst. Sci., 7, 549-556, 2007 ,

http://www.nat-hazards-earth-syst-sci.net/7/549/2007/.

Goldberger, A. L., Peng C.-K., and Lipsitz, L. A.: What is physiologic complexity and how does it change with aging and disease?, Neurobiol. Aging, 23, 23-26, 2002.

Gotoh, K., Hayakawa, M., and Smirnova, N.: Fractal analysis of the ULF geomagnetic data obtained at Izu Peninsula, Japan in relation to the nearby earthquake swarm of JuneAugust 2000, Nat. Hazards Earth Syst. Sci., 3, 229-236, 2003, http://www.nat-hazards-earth-syst-sci.net/3/229/2003/.

Gotoh, K., Hayakawa, M., Smirnova, N. A., and Hattori, K.: Fractal analysis of seismogenic ULF emissions, Phys. Chem. Earth, 29, 419-424, 2004.

Grassberger, P. and Procaccia, I.: Estimation of the Kolmogorov entropy from a chaotic signal, Phys. Rev. A, 28, 2591-2593, 1983.

Hayakawa, M., Ito, T., and Smirnova, N.: Fractal analysis of ULF geomagnetic data associated with the Guam earthquake on $8 \mathrm{Au}-$ gust 1993, Geophys. Res. Lett., 26, 2797-2800, 1999.

Hayakawa, M. (Ed.): Atmospheric and Ionospheric Phenomena Associated with Earthquakes, Terra Sc. Publ. Tokyo, 996 pp., 1999.

Hayakawa, M. and Molchanov, O. A. (Eds.): Seismo Electromagnetics: Lithosphere-Atmosphere-Ionosphere Coupling, Terra Sc. Publ. Tokyo, 2002.

Ida, Y., Hayakawa, M., Adalev, A., and Gotoh, K.: Multifractal analysis for the ULF geomagnetic data during the 1993 Guam earthquake, Nonlin. Processes Geophys., 12, 157-162, 2005, http://www.nonlin-processes-geophys.net/12/157/2005/.

Ida, Y. and Hayakawa, M.: Fractal analysis for the ULF data during the 1993 Guam earthquake to study prefracture criticality, Nonlin. Processes Geophys., 13, 409-412, 2006, http://www.nonlin-processes-geophys.net/13/409/2006/.

Lazaridou, M., Varotsos, C., Alexopoulos, K., and Varotsos, P.: Point defectparameters of LiF, J. Physics C: Solid State, 18, 3891-3895, 1985.

Lomnitz, C.: Fundamentals of Earthquake Prediction, John Wiley \& Sons, New York, 1990.

Makse, H., Havlin, S., Schwartz, M., and Stanley, H. E.: Method for Generating Long-Range Correlations for Large Systems, Phys. Rev. E, 53, 5445-5449, 1996.

Muñoz-Diosdado, A., Pavía-Miller, C. G., Angulo-Brown, F., and Ramírez-Rojas, A.: Spectral and multifractal study of electroseismic time series associated to the $\mathrm{Mw}=6.5$ earthquake of 24 October 1993 in Mexico, Nat. Hazards Earth Syst. Sci., 4, 703709, 2004, http://www.nat-hazards-earth-syst-sci.net/4/703/2004/.

Pincus, S. M.: Approximate entropy as a measure of system complexity, Proc. Natl. Acad. Sci. USA, 88, 2297-2301, 1991.

Pincus, S. M.: Approximate entropy (ApEn) as a complexity measure, Chaos, 5, 110-117, 1995.

Ramírez-Rojas, A., Pavía-Miller, C. G. and Angulo-Brown, F.: Statistical behavior of the spectral exponent and the correlation time of electric self-potential time series associated to the Ms=7.4 September 14, 1995 earthquake in Mexico, Phys. Chem. Earth, 29(4-9), 305-312, 2004.
Ramírez-Rojas, A., Flores-Márquez, E. L., Guzmán-Vargas, L., Márquez-Cruz, J., Pavía-Miller, C. G., and Angulo-Brown, F.: A comparison of ground geoelectric activity between three regions of different level of seismicity, Nat. Hazards Earth Syst. Sci., 7, 591-598, 2007, http://www.nat-hazards-earth-syst-sci.net/7/591/2007/.

Richman, J. S. and Moorman, J. R.: Physiological time-series analysis using approximate entropy and sample entropy, Am. J. Physiol. Heart Circ. Physiol., 278, H2039-49, 2000.

Smirnova, N., Hayakawa, M., and Gotoh, K.: Precursory behavior of fractal characteristics of the ULF electromagnetic fields in seismic active zones before strong earthquakes, Phys. Chem. Earth, 29, 445-451, 2004.

Telesca, L., Cuomo, V., Lapenna, V., and Macchiato, M.: A new approach to investigate the correlation between geoelectrical time fluctuations and earthquakes in a seismic area of southern Italy, Geophys. Res. Lett., 28, 4375-4378, 2001.

Telesca, L., Lapenna, V., and Macciato, M.: Multifractal fluctuations in earthquake related geolectrical signals, New J. Phys., 7, 214-228, 2005.

Telesca, L. and Lapenna, V.: Measuring multifractality in seismic sequences, Tectonophysics, 423, 115-123, 2006.

Varotsos, P. A.: An estimate of the pressure dependence of the dielectric constant inalkali halides, Phys. Status Solidi B, 90, 339343, 1978.

Varotsos, P.: Determination of the dielectric constant of alkali halide mixed crystals, Phys. Status Solidi B, 100, K133-138, 1980.

Varotsos, P. and Alexopoulos, K.: Physical properties of the variations of the electric field of the earth preceding earthquakes I, Tectonophysics, 110, 73-98, 1984a.

Varotsos, P. and Alexopoulos, K.: Physical properties of the variations of the electric field of the earth preceding earthquakes II. Determination of epicenter and magnitude, Tectonophysics, 110, 99-125, 1984b.

Varotsos, P. and Alexopoulos, K.: Physical properties of the variations in the electric field of the earth preceding earthquakes, III, Tectonophysics, 136, 335-339, 1987.

Varotsos, P. A., Sarlis, N. V., and Skordas, E. S.: Attempt to distinguish electric signalsof a dichotomous nature, Phys. Rev. E, 68, 031106, 2003.

Varotsos, P. A., Sarlis, N. V., Skordas, E. S, and Lazaridou, M. S.: Entropy in the natural time domain, Phys. Rev. E, 70, 011106, 2004.

Varotsos, P. A.: The Physics of Seismic Electric Signals, Terra Sc. Publ. Tokyo, 2005.

Varotsos, P. A., Sarlis, N. V., Skordas, E. S., and Lazaridou, M. S.: Fluctuations, under time reversal, of the natural time and the entropy distinguish similar looking electric signals of different dynamics, J. Appl. Phys., 103, 014906, 2008.

Yépez, E., Angulo-Brown, F., Peralta, J. A., Pavía-Miller, C. G., and González-Santos, G.: Electric fields patterns as seismic precursors, Geophys. Res. Lett., 22, 3087-3090, 1995. 